\title{
A randomised, placebo-controlled study of the CXCR2 antagonist AZD5069 in bronchiectasis
}

\author{
Anthony De Soyza ${ }^{1}$, Ian Pavord ${ }^{2}$, J. Stuart Elborn ${ }^{3}$, David Smith ${ }^{4}$, Heather Wray ${ }^{5}$, \\ Margareta Puu ${ }^{5}$, Bengt Larsson ${ }^{5}$ and Robert Stockley ${ }^{6}$
}

Affiliations: ${ }^{1}$ Institute of Cellular Medicine, Newcastle University and Freeman Hospital, Sir William Leech Research Centre, Respiratory Department, Newcastle upon Tyne, UK. ${ }^{2}$ Nuffield Department of Medicine, University of Oxford, Oxford, UK. ${ }^{3}$ Centre for Infection and Immunity, Queens University, Belfast, UK. ${ }^{4}$ Respiratory Research Unit, Southmead Hospital, Bristol, UK. ${ }^{5}$ Innovative Medicines, Respiratory, Inflammation and Autoimmunity (iMED RIA), AstraZeneca R\&D, Mölndal, Sweden. ${ }^{6}$ Respiratory Department, Queen Elizabeth Hospital, Birmingham, UK

Correspondence: Anthony De Soyza, Institute of Cellular Medicine, Newcastle University and Sir William Leech Research Centre, Freeman Road, High Heaton, Newcastle upon Tyne, Tyne and Wear, NE7 7DN, UK.

E-mail: anthony.de-soyzaancl.ac.uk

ABSTRACT This randomised double-blind placebo-controlled parallel-group multicentre phase IIa study evaluated the effect of the CXCR2 antagonist AZD5069 on sputum neutrophil counts in adults with bronchiectasis.

Patients were randomised 1:1 to receive AZD5069 $80 \mathrm{mg}$ or placebo orally twice daily for 28 days. Assessments included blood cell counts, inflammatory markers in blood, morning spontaneous sputum, lung function, safety and tolerability and patients completed daily BronkoTest diary cards. The primary outcome measure was the change in absolute sputum neutrophil count.

Of 52 randomised patients, 45 completed treatment, 20 (76.9\%) out of 26 receiving AZD5069 and 25 (96.2\%) out of 26 receiving placebo. AZD5069 reduced the absolute neutrophil cell count in morning sputum by $69 \%$ versus placebo $(\mathrm{p}=0.004)$; percentage sputum neutrophil count was reduced by $36 \%$ $(\mathrm{p}=0.008)$. The number of infections/exacerbations was similar with AZD5069 and placebo (nine versus eight), but these led to more study discontinuations with AZD5069 (four versus zero). Sputum interleukin (IL)- 6 and growth-regulated oncogene (GRO)- $\alpha$ and serum GRO- $\alpha$, IL- $1 ß$ and IL- 8 levels increased with AZD5069 versus placebo (all $\mathrm{p}<0.001$ ), while serum high-sensitivity C-reactive protein levels did not change. AZD5069 was well tolerated.

AZD5069 markedly reduced absolute sputum neutrophil counts in bronchiectasis patients, although this was not associated with improvements in clinical outcomes in this exploratory study.

@ERSpublications

CXCR2 antagonist AZD5069 reduced sputum neutrophil counts in patients with bronchiectasis over 4 weeks of treatment http://ow.ly/OrvSE

Editorial comment in Eur Respir J 2015; 46: 898-900 [DOI: 10.1183/09031936.00961-2015].

This article has supplementary material available from erj.ersjournals.com

Received: Jan 272015 | Accepted after revision: May 282015 | First published online: Sept 042015

Clinical trials: This study is registered at clinicaltrials.gov with identifier number NCT01255592.

Support statement: This study was funded by AstraZeneca. A. De Soyza acknowledges a Higher Education Funding Council for England clinical senior lectureship and support from the Newcastle University National Institute for Health Research Biomedical Research Centre in Ageing. Funding information for this article has been deposited with FundRef.

Conflict of interest: Disclosures can be found alongside the online version of this article at erj.ersjournals.com

Copyright @ERS 2015 


\section{Introduction}

Bronchiectasis is a chronic condition, characterised by dilatation of the airways and neutrophilic airway inflammation, which is associated with frequent exacerbations and/or continuous bacterial colonisation [ 1 , 2]. Current management is largely based on cystic fibrosis/chronic obstructive pulmonary disease (COPD) treatments and includes chest physiotherapy and pharmacotherapy such as antibiotics, anti-inflammatories (inhaled steroids) and other inhaled therapies (bronchodilators), mucolytics, oxygen and noninvasive ventilation $[1,3,4]$. However, these have limited effects $[1,3]$.

Inhaled corticosteroids (ICS) are more effective when inflammation is predominantly eosinophilic [5] rather than neutrophilic, and are not recommended in bronchiectasis unless there is an asthmatic component [3,6-10]. Although ICS induce eosinophil apoptosis and phagocytic removal, they inhibit these processes in neutrophils [7, 11]. In bronchiectasis caused by cystic fibrosis, ICS have been shown to be ineffective [12]. This creates a significant unmet need for new treatment modalities for these conditions, which collectively are responsible for significant healthcare resource utilisation and costs $[1,4,5,9,13,14]$. Bronchiectasis itself has been estimated to cost $>£ 30$ million in hospital admissions in the UK alone [1].

Neutrophil migration into the lungs is regulated by a variety of mediators, most notably interleukin (IL)-8, IL-1 $\beta$, tumour necrosis factor (TNF)- $\alpha$, and leukotriene $B_{4}\left(\mathrm{LTB}_{4}\right)$ [9]. IL-8 is able to bind to several surface membrane receptors, including cysteine-X-cysteine (CXC) chemokine receptor-2 (CXCR2), which is expressed on neutrophils, epithelial cells and other cells involved in bronchial inflammation $[9,15,16]$. CXCR2 antagonists have been shown to reduce the inflammatory neutrophilic response in the lung and nose to ozone or inhaled lipopolysaccharide challenge $[9,17-20]$.

AZD5069 is a selective and reversible CXCR2 antagonist that is predicted to reduce neutrophil migration from bone marrow into the bloodstream and from the systemic circulation into other compartments, such as the lungs. AZD5069 reduces bronchial mucosal neutrophil infiltration in animal models (data on file; AstraZeneca, 2014). In this phase IIa proof-of-concept study, the effects of an oral dose of AZD5069 were evaluated in bronchiectasis patients for the first time. We tested the hypothesis that AZD5069 reduces the elevated sputum neutrophil count characteristic of bronchiectasis.

\section{Materials and methods}

\section{Study design}

This was a randomised double-blind placebo-controlled parallel-group phase IIa study in 52 adult patients with bronchiectasis conducted at 16 sites (online supplementary material) in the UK, Poland and the Czech Republic (clinicaltrials.gov identifier number NCT01255592). Patients were enrolled after they had provided informed consent. A BronkoTest (BronkoTest Ltd, London, UK) diary card was issued at visit 1 and patients were instructed to complete it daily throughout the study, recording sputum colour in addiiton, and bring it for review and assessment at each clinic visit. The St George's Respiratory Questionnaire for COPD patients (SGRQ-C), validated for use in bronchiectasis [21, 22], was administered at clinic visits, along with assessment of the transition dyspnoea index (TDI). Other clinic visit methodologies are detailed in the online supplementary material. The treatment period started at visit 2 (day 1) when subjects were randomised 1:1 to receive either AZD5069 $80 \mathrm{mg}$ or placebo as capsules orally twice daily for 28 days (fig. 1).

\section{Study subjects}

The study population consisted of females of non-childbearing potential and males with a clinical diagnosis of idiopathic or post-infective bronchiectasis confirmed by high-resolution computed tomography scan or bronchogram (online supplementary material). All had a history of sputum production with chronic expectoration on most days of most weeks in the year and were able to provide at least two of the three required baseline sputum samples (mean $\geqslant 3 \mathrm{~mL}$ ).

\section{Interventions}

Patients were randomised 1:1 on visit 2 (day 1) to receive either AZD5069 $80 \mathrm{mg}$ or placebo as capsules orally twice daily for $28 \pm 2$ days. The dose selected was based on previous pharmacokinetic/pharmacodynamic assessments from healthy volunteer studies which predicted that this dose would have maximum effects on sputum neutrophil counts while avoiding lowering blood neutrophil counts for prolonged periods [23] (data on file; AstraZeneca, 2014). There was a predefined study withdrawal criterion for blood neutrophils $<1.0 \times 10^{9}$ cells $\cdot \mathrm{L}^{-1}$ observed at least twice during a 48 -h period. Compliance was assessed by tablet counts.

\section{Study end-points}

The primary end-point was the change in absolute neutrophil cell count in morning 2-h spontaneous sputum samples. Secondary end-points included the percentage neutrophil cell count in sputum, signs and 


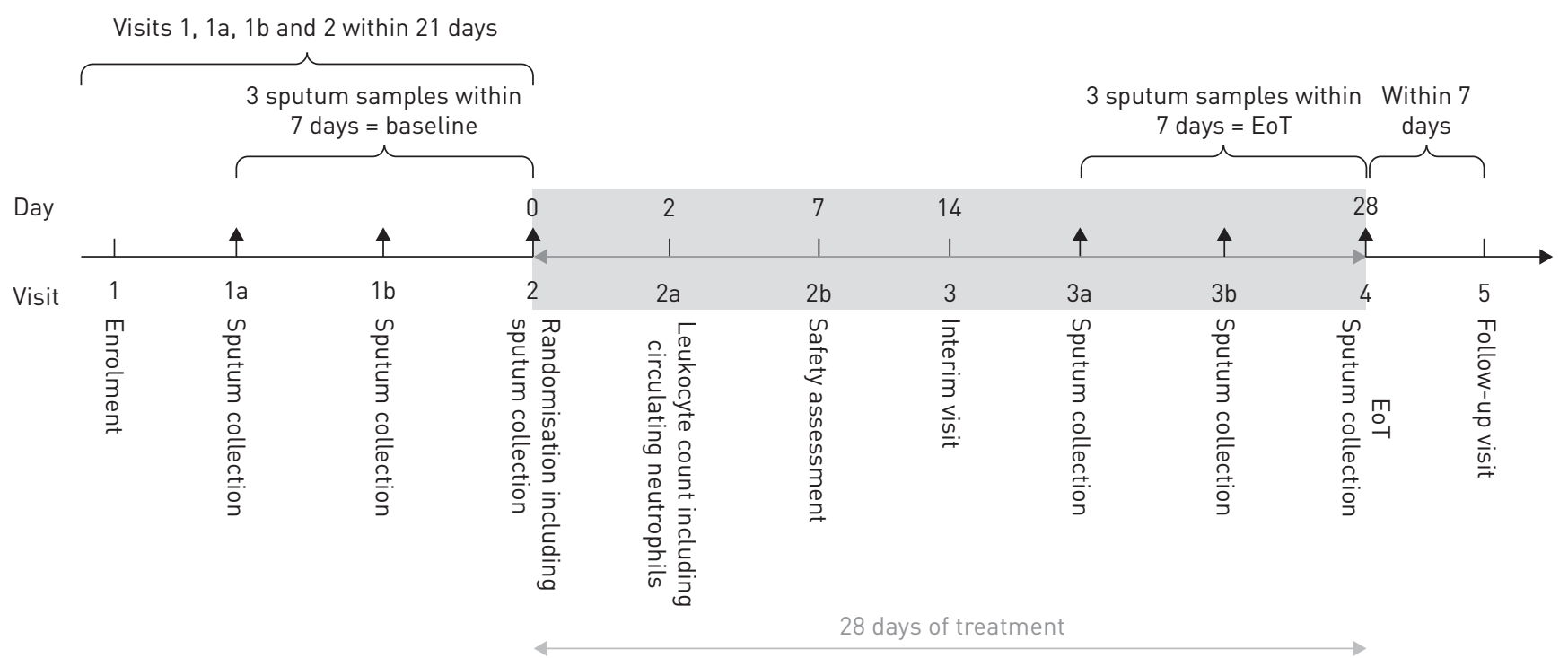

FIGURE 1 Flow chart of study design. EoT: end of treatment.

symptoms of bronchiectasis, lung function and dyspnoea (based on the BronkoTest diary cards). Health-related quality of life (HRQoL) was assessed using the SGRQ-C. Safety and tolerability assessments included laboratory and clinical assessments, physical examination, 12-lead ECG and recorded adverse events over the 28 days of the study and, in particular, exacerbations, as defined by the criteria defined by Anthonisen et al. [24]. Because reductions in neutrophil count may be associated with an increased risk of infection, patients were regularly monitored for this [25]. The effect of AZD5069 on other inflammatory markers in sputum and blood was also assessed. Sputum IL-1 $\beta$, IL-6, chemokine (C-C motif) ligand 5 or RANTES, monocyte chemotactic protein-1, TNF- $\alpha$, growth-regulated oncogene (GRO)- $\alpha$ and IL- 8 were assayed as a priority. Neutrophil elastase activity was assessed when sufficient sputum volume remained. The selected serum markers of inflammation were high-sensitivity C-reactive protein (hsCRP), TNF- $\alpha$, GRO- $\alpha$, IL-6, IL-1 $\beta$, IL-8 and amyloid-A.

\section{Statistical analyses}

Data analyses were based on patient sets. The "all patients analysis" set comprised all patients who were screened for the study and has been used for reporting of disposition and screening failures. The other sets consisted of all patients who were randomised and received at least one dose of the study medication. The "efficacy analysis" set, based on the intention-to-treat principle included all patients who contributed data for at least one efficacy end-point; the "safety analysis" set consisted of patients who had data collected after randomisation, while the "pharmacodynamics analysis" set comprised all patients for whom pharmacodynamics samples (absolute and percentage neutrophil cell count in sputum, sputum collection weight, inflammatory markers in sputum and serum) were available.

A sample size of 22 patients in each treatment group was calculated to have $78 \%$ power to detect the hypothesised reduction of 50\% in sputum neutrophils for AZD5069 compared with placebo, assuming a standard deviation of 0.93 and a one-sided t-test at a 5\% significance level for the comparison of two population means.

All hypothesis testing was conducted using two-sided tests and $\mathrm{p}<0.10$ values were considered statistically significant [26]. All p-values presented are two sided. Where a baseline variable was missing, the value recorded at a different pre-treatment visit was substituted. A decision on the strategy to deal with any missing variable was documented prior to unblinding of the treatment codes.

\section{Randomisation and masking}

Patients were assigned to treatment groups using interactive voice and web response systems (IVRS/IWRS). Routines for this were described in the IVRS/IWRS user manual provided to each site (see the online supplementary material for further details). Randomisation was stratified for use of ICS (yes/no) and Pseudomonas aeruginosa infection (past or current). Patients were randomly allocated to AZD5069 $80 \mathrm{mg}$ twice daily, provided as a capsule for oral administration, or matching placebo in a 1:1 ratio. Individual treatment codes, indicating the treatment randomisation for each randomised patient, were available to the 
investigator(s) or pharmacists from the IVRS/IWRS. The treatment code was not to be broken except in medical emergencies when the appropriate management of the patient required knowledge of the treatment randomisation. To avoid unblinding site and study staff, blood leukocyte counts, including neutrophil data, were sent directly from local laboratories to the central study laboratory, which reviewed the data and notified the site if a patient needed to be recalled for repeat sampling or withdrawn from the study.

\section{Results}

Study subjects

From 83 patients who enrolled in the study, 52 were randomised to AZD5069 or placebo (fig. 2). The first patient was enrolled December 27, 2010 and the last patient last visit was February 13, 2012. The mean age of the randomised patients was 65 years (range 34-79 years) and mean forced expiratory volume in $1 \mathrm{~s}$ $\left(F_{1}\right)$ on entry was $1.70 \mathrm{~L}$ (table 1). All of the patients were white Caucasians and $54 \%$ were female. Of the 52 patients randomised, 38 were from the UK, 10 from Poland, and four from the Czech Republic. Mean duration of known bronchiectasis was 17.5 years. Bronchiectasis symptoms at baseline were assessed and classified by investigators as purulent sputum ( $P$. aeruginosa culture positive) $19.2 \%$, purulent sputum (P. aeruginosa negative) $63.5 \%$ and mucoid sputum $15.4 \%$.

The two treatment groups were generally similar in terms of age, race, weight, height, body mass index, bronchiectasis type, nicotine use and concomitant medication use (table 1). However, despite randomisation the AZD5069 group differed from the placebo group in the proportion of females $(61.5 \%$ versus $46.2 \%$ ), longer mean duration of bronchiectasis (22 versus 13 years) and a greater number of neutrophils in their sputum at baseline (mean 5.5 versus $3.9 \times 10^{6} \mathrm{cells}^{-1} \mathrm{~g}^{-1}$ ). Lung function at baseline was slightly lower in the AZD5069 versus the placebo group (mean FEV1 $1.52 \mathrm{~L}$ versus $1.88 \mathrm{~L}$ ).

In total, 45 (86.5\%) out of 52 patients completed treatment and the study; 20 (76.9\%) out of 26 patients in the AZD5069 group and $25(96.2 \%)$ out of 26 in the placebo group. In the AZD5069 group, five (19.2\%) patients withdrew due to adverse events and one withdrew consent. In the placebo group, one (3.8\%) patient discontinued due to other reasons (fig. 2).

Patients who received AZD5069 had a mean (range) compliance of 98.1\% (89.3-123.1\%) and patients who received placebo had a mean (range) compliance of $97.7 \%$ (87.1-100.0\%). All patients were $>80 \%$ compliant with their study medication.

\section{Efficacy evaluations}

Absolute sputum neutrophil cell count was reduced by $69 \%$ after treatment with AZD5069 versus placebo (ratio $0.31 ; 90 \%$ CI $0.17-0.59$ ) using the pharmacodynamics analysis set ( $p=0.004$ ) (fig. 3 and online supplementary table S1a and b). In the placebo-treated group, absolute mean sputum neutrophil counts

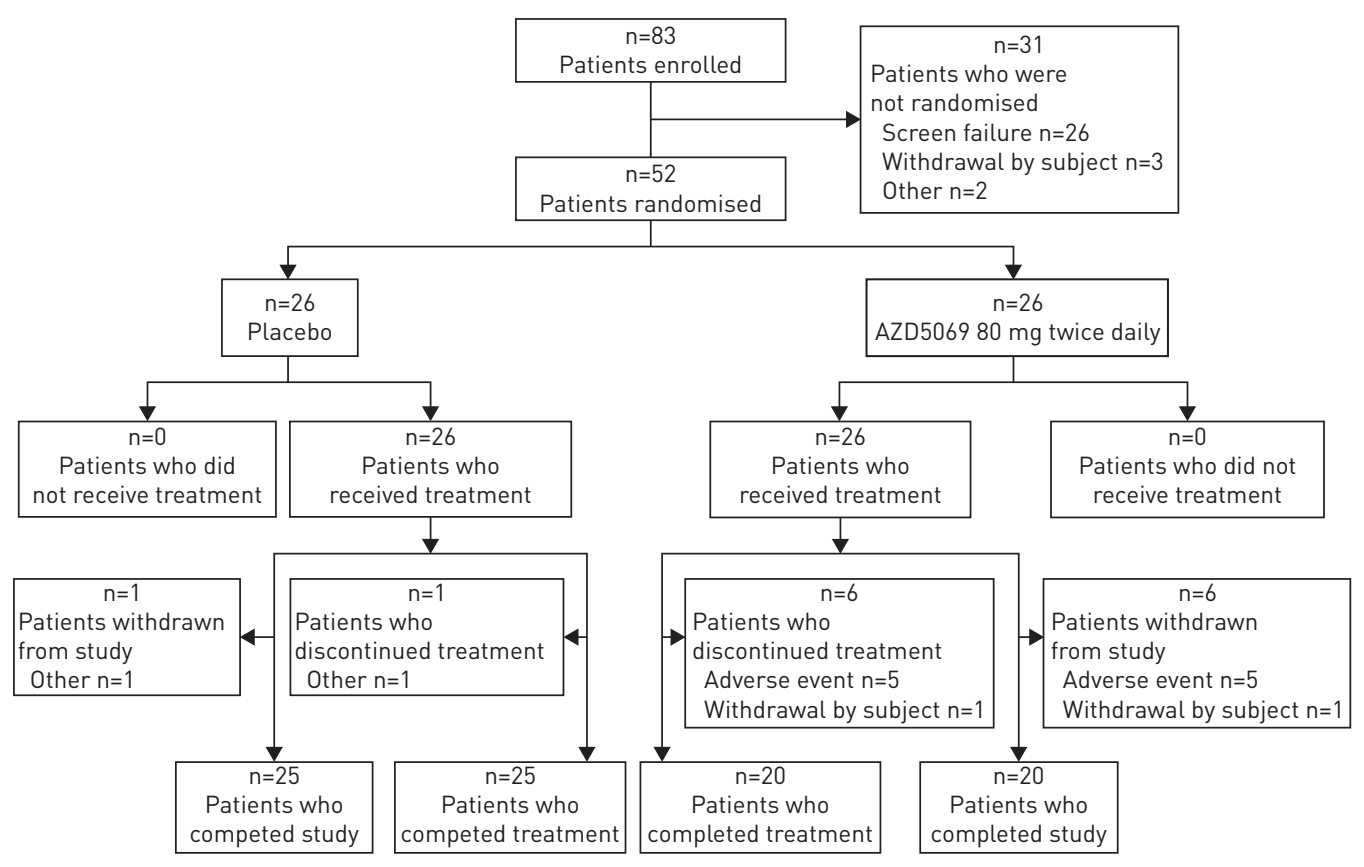

FIGURE 2 Patient disposition. 
TABLE 1 Demographic and other patient characteristics: safety analysis set

\begin{tabular}{|c|c|c|c|}
\hline & Placebo & AZD5069 & Total \\
\hline Subjects $n$ & 26 & 26 & 52 \\
\hline Age years & $65 \pm 8.8$ & $66 \pm 6.6$ & $65 \pm 7.7$ \\
\hline Female & $12(46.2)$ & $16(61.5)$ & $28(53.8)$ \\
\hline White Caucasian & $26(100)$ & $26(100)$ & $52(100)$ \\
\hline Weight kg & $77 \pm 17.0$ & $75 \pm 15.8$ & $76 \pm 16.3$ \\
\hline Height $\mathrm{cm}$ & $168 \pm 9.6$ & $166 \pm 8.3$ & $167 \pm 8.9$ \\
\hline $\mathrm{BMI} \mathbf{k g} \cdot \mathrm{m}^{-2}$ & $27.4 \pm 6.2$ & $26.7 \pm 4.1$ & $27.1 \pm 5.2$ \\
\hline Duration of bronchiectasis" years & $13 \pm 21.8$ & $22 \pm 22.9$ & $17.5 \pm 22.6$ \\
\hline \multicolumn{4}{|l|}{ Type of bronchiectasis } \\
\hline Purulent sputum (Pseudomonas aeruginosa) & $4(15.4)$ & $6(23.1)$ & $10(19.2)$ \\
\hline Purulent sputum (non-Pseudomonas aeruginosa) & $16(61.5)$ & $17(65.4)$ & 33 (63.5) \\
\hline Mucoid sputum & $5(19.2)$ & $3(11.5)$ & $8(15.4)$ \\
\hline Lung function (FEV 1 on day 1 ) $L$ & $1.88 \pm 0.96$ & $1.52 \pm 0.54$ & 1.70 \\
\hline \multicolumn{4}{|l|}{ Nicotine use } \\
\hline Never & 14 (53.8) & $12(46.2)$ & $26(50.0)$ \\
\hline Former & $10(38.5)$ & 10 (38.5) & 20 (38.5) \\
\hline Current & $2(7.7)$ & $4(15.4)$ & $6(11.5)$ \\
\hline \multicolumn{4}{|l|}{ Concomitant medications ${ }^{\pi}$} \\
\hline Salbutamol & $14(53.8)$ & $17(65.4)$ & $31(59.6)$ \\
\hline Fluticasone + salmeterol & $9(34.6)$ & $9(34.6)$ & $18(34.6)$ \\
\hline Paracetamol & $1(3.8)$ & 8 (30.8) & 9 (17.3) \\
\hline Carbocisteine & $5(19.2)$ & $6(23.1)$ & $11(21.2)$ \\
\hline Budesonide + formoterol & $2(7.7)$ & $5(19 \cdot 2)$ & $7(13.5)$ \\
\hline Ramipril & 1 (3.8) & $5(19.2)$ & $6(11.5)$ \\
\hline Tiotropium bromide & $2(7.7)$ & $4(15.4)$ & $6(11.5)$ \\
\hline Tiotropium & $5(19.2)$ & $3(11.5)$ & $8(15.4)$ \\
\hline Omeprazole & $6(23.1)$ & $4(15.4)$ & 10 (19.2) \\
\hline Azithromycin & $3(11.5)$ & $4(15.4)$ & $7(13.5)$ \\
\hline Acetylsalicylic acid & $6(23.1)$ & $2(7.7)$ & $8(15.4)$ \\
\hline
\end{tabular}

were virtually unchanged at the end of the treatment period (ratio of end of treatment to baseline values was 1.044 for placebo versus 0.297 for AZD5069). There was a 36\% reduction in percentage sputum neutrophil counts with AZD5069 versus placebo (ratio 0.64; 90\% CI 0.49-0.84), which was also statistically significant $(p=0.008)$. The mean percentage sputum neutrophil counts remained consistent over time in the placebo group.

There was a mean difference of $6.78 \mathrm{~g}(90 \%$ CI $1.22-12.33)$ in 24 -h sputum weights $(\mathrm{p}=0.047)$ between treatment groups by the end of the study, with a mean decrease from baseline to end of treatment of $3.3 \mathrm{~g}$ in the placebo group and a mean increase of sputum expectoration of $3.5 \mathrm{~g}$ in the AZD5069 group. There were no significant effects on spirometry (e.g. FEV1 and forced vital capacity), TDI, symptom scores derived from BronkoTest diary cards or SGRQ-C for AZD5069 compared with placebo (fig. 4).

In the exploratory analyses for markers of inflammation, the sputum data showed an estimated 4.5-fold increase in IL-6 and 3.2-fold increase in GRO- $\alpha$ after AZD5069 versus placebo (both $\mathrm{p}<0.001$ ) (table 2). Analysis of serum data indicated a 5.5-fold increase in GRO- $\alpha(p<0.001)$, 1.6-fold increase in IL-1ß $(\mathrm{p}=0.001)$, and a six-fold increase in IL-8 $(\mathrm{p}<0.001)$ (table 3$)$. There were no significant concomitant increases in blood hsCRP.

\section{Safety evaluations}

Overall, 23 (88.4\%) patients receiving AZD5069 and 16 (61.5\%) patients taking placebo reported at least one adverse event during treatment. In the AZD5069 group, six patients discontinued: five (19.2\%) patients discontinued due to adverse events (online supplementary table S2), one of which was classed as a serious adverse event (SAE), and one (3.8\%) patient withdrew consent. The SAE was an episode of pneumonia that required hospitalisation and the patient discontinued the study, although the investigator 

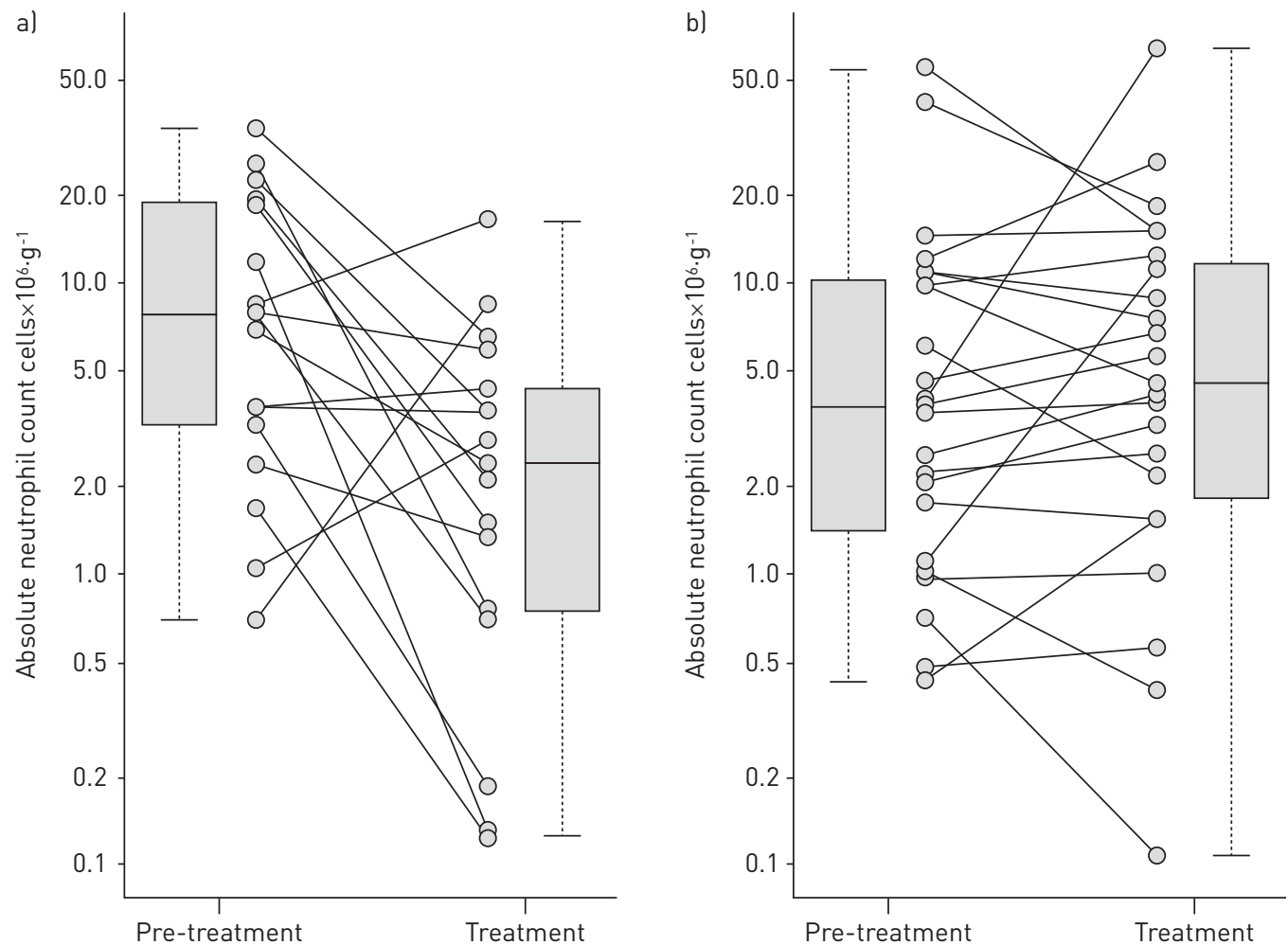

FIGURE 3 Changes in sputum neutrophils in the a) AZD5069 $80 \mathrm{mg}$ twice daily and b) placebo groups. A $69 \%$ reduction of sputum neutrophils was seen in the AZD5069 group compared with placebo. Baseline was calculated as the geometric mean of results obtained at visits 1a, 1b and 2. End of treatment was calculated as the geometric mean of results obtained at visits $3 a, 3 b$ and 4 . The expected improvement is a decrease in absolute neutrophil counts. One-sided $\mathrm{p}=0.002$.

considered that these events were not related to study medication. Three out of the other four discontinuations were also because of exacerbations of bronchiectasis that did not require hospitalisation.

There were no SAEs or discontinuations due to adverse events reported with placebo. In the placebo group, one (3.8\%) patient discontinued from the study as a result of other reasons. Infections were the most reported adverse events by system organ class for both the AZD5069 (nine patients, 34.6\%) and placebo (eight patients, 30.8\%) treatment groups (table 4). The most commonly reported adverse events were cough, headache and rhinorrhoea with AZD5069 and nasopharyngitis with placebo.

Clinical laboratory assessments showed a slight increase in platelets with AZD5069 that remained within the normal reference range. There were no other notable changes, other than the expected decreases in blood leukocytes and neutrophils, which had returned to baseline levels by follow-up. The predefined stopping criterion for blood neutrophil counts, a reduction to $<1 \times 10^{9}$ cells. $\mathrm{L}^{-1}$ observed at least twice over $48 \mathrm{~h}$ did not occur in any individual patient during the study. Clinical chemistry analyses and urinalysis were all unremarkable.

There were no clinically relevant changes in vital signs or ECG. No changes in physical findings on examination were reported.

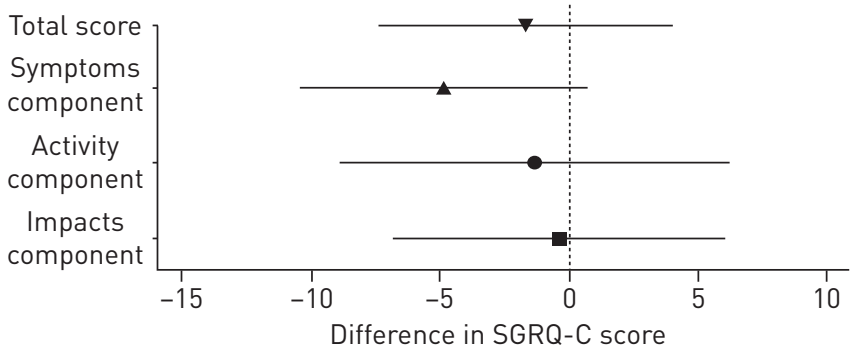

FIGURE 4 Change in St George's Respiratory Questionnaire for chronic obstructive pulmonary disease patients (SGRQ-C) total and domain scores from baseline to end of treatment (difference $190 \% \quad \mathrm{Cl})$ between AZD5069 $80 \mathrm{mg}$ twice daily and placebo). Difference and $90 \% \mathrm{Cl}$ were obtained from an ANCOVA including terms for treatment, inhaled corticosteroids, Pseudomonas aeruginosa infection and baseline. 


\begin{tabular}{|c|c|c|c|c|c|c|c|c|c|c|c|}
\hline & \multirow{2}{*}{\multicolumn{2}{|c|}{ Baseline }} & \multirow{2}{*}{\multicolumn{4}{|c|}{ End of treatment }} & \multicolumn{4}{|c|}{ ANCOVA } & \multirow[t]{3}{*}{ p-value } \\
\hline & & & & & & & \multicolumn{2}{|c|}{$\begin{array}{l}\text { Ratio of end of } \\
\text { treatment to baseline }\end{array}$} & \multicolumn{2}{|c|}{$\begin{array}{c}\text { Ratio of AZD5069 } \\
80 \mathrm{mg} \text { twice daily to placebo }\end{array}$} & \\
\hline & Subjects $n$ & Geo mean & CV \% & Subjects $n$ & Geo mean & CV \% & Subjects $\mathrm{n}$ & Ratio $(90 \% \mathrm{CI})$ & Subjects $\mathbf{n}$ & Ratio $(90 \%$ CI) & \\
\hline \multicolumn{12}{|l|}{$\mathrm{IL}-1 \beta \mathrm{pg} \cdot \mathrm{mL}^{-1}$} \\
\hline Placebo & 25 & 924.7 & 318.6 & 25 & 863.2 & 424.5 & 25 & $0.9(0.7-1.3)$ & & & \\
\hline AZD5069 & 22 & 1384.3 & 319.5 & 22 & 847.2 & 357.3 & 22 & $0.6(0.4-0.9)$ & 47 & $0.7(0.4-1.1)$ & 0.220 \\
\hline \multicolumn{12}{|l|}{ IL-6 pg.mL $\mathrm{mL}^{-1}$} \\
\hline Placebo & 25 & 122.8 & 163.4 & 25 & 108.4 & 155.2 & 25 & $0.8(0.6-1.1)$ & & & \\
\hline AZD5069 & 22 & 193.0 & 216.5 & 22 & 655.8 & 152.4 & 22 & $3.7(2.8-4.8)$ & 47 & $4.5(3.1-6.5)$ & $<0.001$ \\
\hline \multicolumn{12}{|c|}{ RANTES $\mathrm{pg} \cdot \mathrm{mL}^{-1}$} \\
\hline Placebo & 25 & 19.8 & 132.1 & 25 & 20.9 & 124.8 & 25 & $1.1(0.9-1.3)$ & & & \\
\hline AZD5069 & 22 & 18.0 & 97.4 & 22 & 18.1 & 113.9 & 22 & $1.0(0.8-1.2)$ & 47 & $0.9(0.7-1.3)$ & 0.670 \\
\hline \multicolumn{12}{|l|}{ MCP-1 pg $\cdot \mathrm{mL}^{-1}$} \\
\hline Placebo & 25 & 600.8 & 106.8 & 25 & 570.9 & 77.4 & 25 & $0.9(0.8-1.1)$ & & & \\
\hline AZD5069 & 22 & 685.2 & 74.0 & 22 & 603.6 & 55.4 & 22 & $0.9(0.8-1.1)$ & 47 & $1.0(0.8-1.3)$ & 0.968 \\
\hline \multicolumn{12}{|l|}{ TNF- $\alpha \mathrm{pg} \cdot \mathrm{mL}^{-1}$} \\
\hline Placebo & 25 & 48.2 & 489.5 & 25 & 43.6 & 884.2 & 25 & $1.0(0.7-1.2)$ & & & \\
\hline AZD5069 & 22 & 67.7 & 393.9 & 22 & 87.9 & 452.5 & 22 & $1.3(0.9-1.8)$ & 47 & $1.4(0.9-2.2)$ & 0.193 \\
\hline \multicolumn{12}{|l|}{$\mathrm{GRO}-\alpha \mathrm{pg} \cdot \mathrm{mL}^{-1}$} \\
\hline Placebo & 25 & 8170.2 & 487.4 & 25 & 7722.8 & 277.8 & 25 & $0.9(0.7-1.1)$ & & & \\
\hline AZD5069 & 22 & 13883.9 & 276.4 & 22 & 34228.8 & 152.6 & 22 & $2.8(2.1-3.7)$ & 47 & $3.2(2.2-4.8)$ & $<0.001$ \\
\hline \multicolumn{12}{|l|}{$\mathrm{IL}-8 \mathrm{pg} \cdot \mathrm{mL}^{-1}$} \\
\hline Placebo & 26 & 16682.3 & 162.7 & 26 & 13362.1 & 236.8 & 26 & $0.8(0.6-1.0)$ & & & \\
\hline AZD5069 & 22 & 19924.9 & 170.7 & 22 & 16116.9 & 162.1 & 22 & $0.8(0.6-1.1)$ & 48 & $1.0(0.7-1.5)$ & 0.917 \\
\hline \multicolumn{12}{|c|}{ Neutrophil elastase activity $\mu \mathrm{mol} \cdot \mathrm{L}^{-1} \cdot \mathrm{h}^{-1}$} \\
\hline Placebo & 5 & 11.9 & 35745.8 & 5 & 19.6 & 131038.6 & 5 & $2.0(0.6-6.4)$ & & & \\
\hline AZD5069 & 4 & 2.9 & 775.1 & 4 & 1.2 & 239.9 & 4 & $0.3(0.1-1.3)$ & 9 & $0.2(0.0-1.1)$ & 0.111 \\
\hline
\end{tabular}

Geo mean: geometric mean; CV: coefficient of variation; IL: interleukin; MCP: monocyte chemotactic protein; TNF: tumour necrosis factor; GRO: growth-regulated oncogene. 
TABLE 3 Analysis of the serum inflammatory marker data: ratio of AZD5069 $80 \mathrm{mg}$ twice daily to placebo in the pharmacodynamics analysis set

\begin{tabular}{|c|c|c|c|c|c|c|c|c|c|c|c|}
\hline & \multirow{2}{*}{\multicolumn{2}{|c|}{ Baseline }} & \multirow{2}{*}{\multicolumn{4}{|c|}{ End of treatment }} & \multicolumn{4}{|c|}{ ANCOVA } & \multirow[t]{3}{*}{ p-value } \\
\hline & & & & & & & \multicolumn{2}{|c|}{$\begin{array}{l}\text { Ratio of end of } \\
\text { treatment to baseline }\end{array}$} & \multicolumn{2}{|c|}{$\begin{array}{l}\text { Ratio of AZD5069 } 80 \mathrm{mg} \\
\text { twice daily to placebo }\end{array}$} & \\
\hline & Subjects $n$ & Geo mean & CV \% & Subjects n & Geo mean & CV \% & Subjects $n$ & Ratio $(90 \% \mathrm{CI})$ & Subjects $n$ & Ratio $(90 \% \mathrm{Cl})$ & \\
\hline \multicolumn{12}{|l|}{ CRP $\mathrm{mg} \cdot \mathrm{L}^{-1}$} \\
\hline Placebo & 24 & 6.3 & 185.6 & 24 & 5.1 & 228.2 & 24 & $0.8(0 \cdot 6-1.1)$ & & & \\
\hline AZD5069 & 24 & 5.8 & 130.4 & 24 & 7.7 & 120.1 & 24 & $1.3(0.9-1.8)$ & 48 & $1.5(1.0-2.4)$ & 0.112 \\
\hline \multicolumn{12}{|l|}{$\mathrm{IL}-8 \mathrm{pg} \cdot \mathrm{mL}^{-1}$} \\
\hline Placebo & 24 & 16.6 & 45.5 & 24 & 15.3 & 58.2 & 24 & $1.0(0.8-1.2)$ & & & \\
\hline AZD5069 & 24 & 12.9 & 36.4 & 24 & 80.2 & 83.9 & 24 & $5.9(4.7-7.3)$ & 48 & $6.1(4.4-8.4)$ & $<0.001$ \\
\hline \multicolumn{12}{|l|}{$\mathrm{IL}-6 \mathrm{pg} \cdot \mathrm{mL}^{-1}$} \\
\hline Placebo & 24 & 3.4 & 104.8 & 24 & 3.2 & 97.5 & 24 & $1.0(0.8-1.2)$ & & & \\
\hline AZD5069 & 24 & 2.3 & 109.0 & 24 & 3.2 & 88.3 & 24 & $1.1(1.0-1.3)$ & 48 & $1.2(0.9-1.5)$ & 0.281 \\
\hline \multicolumn{12}{|l|}{$\mathrm{IL}-1 \beta \mathrm{pg} \cdot \mathrm{mL}^{-1}$} \\
\hline Placebo & 24 & 0.7 & 64.5 & 24 & 0.6 & 74.2 & 24 & $0.9(0.8-1.1)$ & & & \\
\hline AZD5069 & 24 & 0.6 & 88.2 & 24 & 0.9 & 89.2 & 24 & $1.5(1.3-1.7)$ & 48 & $1.6(1.3-1.9)$ & 0.001 \\
\hline \multicolumn{12}{|l|}{$\mathrm{GRO}-\alpha \mathrm{pg} \cdot \mathrm{mL}^{-1}$} \\
\hline Placebo & 24 & 146.4 & 43.8 & 24 & 146.1 & 48.6 & 24 & $1.0(0.8-1.2)$ & & & \\
\hline AZD5069 & 16 & 156.9 & 37.5 & 16 & 846.8 & 91.8 & 16 & $5.5(4.4-6.7)$ & 40 & $5.5(4.2-7.2)$ & $<0.001$ \\
\hline \multicolumn{12}{|l|}{ TNF- $\alpha \mathrm{pg} \cdot \mathrm{mL}^{-1}$} \\
\hline Placebo & 24 & 9.4 & 26.9 & 24 & 8.9 & 17.9 & 24 & $1.0(0.9-1.0)$ & & & \\
\hline AZD5069 & 24 & 8.7 & 34.7 & 24 & 9.0 & 23.5 & 24 & $1.0(1.0-1.1)$ & 48 & $1.1(1.0-1.1)$ & 0.241 \\
\hline \multicolumn{12}{|l|}{$\mathrm{SAA} n \mathrm{ng} \cdot \mathrm{mL}^{-1}$} \\
\hline Placebo & 24 & 9924.2 & 243.7 & 24 & 8594.3 & 300.0 & 24 & $0.9(0.6-1.3)$ & & & \\
\hline AZD5069 & 24 & 8865.5 & 209.8 & 24 & 10781.3 & 230.2 & 24 & $1.2(0.8-1.7)$ & 48 & $1.3(0.8-2.3)$ & 0.367 \\
\hline
\end{tabular}


TABLE 4 Adverse events reported during treatment, by system organ class: safety analysis set

\begin{tabular}{lcc} 
& AZD5069 $\mathbf{8 0 ~} \mathbf{~ m g ~ t w i c e ~ d a i l y ~}$ & Placebo \\
\hline Subjects & 26 & 26 \\
Patients with any adverse event & $23(88.5)$ & $16(61.5)$ \\
Infections and infestations & $9(34.6)$ & $8(30.8)$ \\
Respiratory, thoracic and mediastinal disorders & $9(34.6)$ & $5(19.2)$ \\
Nervous system disorders & $7(26.9)$ & $6(23.1)$ \\
Gastrointestinal disorders & $6(23.1)$ & $4(15.4)$ \\
General disorders and administration-site conditions & $5(19.2)$ & $3(11.5)$ \\
Musculoskeletal and connective tissue disorders & $3(11.5)$ & $5(19.2)$ \\
Investigations & $3(11.5)$ & $2(7.7)$ \\
Renal and urinary disorders & $2(7.7)$ & $1(3.8)$ \\
Skin and subcutaneous tissue disorders & $2(7.7)$ & 0 \\
Ear and labyrinth disorders & $1(3.8)$ & $2(7.7)$ \\
Injury, poisoning and procedural complications & $1(3.8)$ & 0 \\
Psychiatric disorders & $1(3.8)$ & 0 \\
Eye disorders & 0 & $2(7.7)$ \\
Metabolism and nutrition disorders & 0 & $1(3.8)$ \\
\hline
\end{tabular}

Data are presented as $\mathrm{n}$ or $\mathrm{n}(\%)$. Patients who had at least one adverse event in any system organ class, presented in order of decreasing frequency (sorted by total number on AZD5069 $80 \mathrm{mg}$ twice daily) using MedDRA (version 14.1; www.meddra.org).

\section{Discussion}

There is a pressing need to reduce morbidity and mortality in bronchiectasis. Currently there are no therapies licensed for bronchiectasis as an indication $[1,9,14]$. Targeting excessive neutrophilic inflammation is an attractive option in bronchiectasis. In this randomised double-blind placebo-controlled study in adult patients with bronchiectasis, there was a statistically significant $69 \%$ reduction in absolute neutrophil cell count in the sputum of the bronchiectasis patients receiving AZD5069 compared with placebo. To our knowledge such a dramatic fall in sputum neutrophilia has not been described in bronchiectasis before.

The tolerability of AZD5069 was generally good as reported in previous studies $[27,28]$. Although a higher proportion of patients on AZD5069 had adverse events compared with placebo, the adverse events were mostly minor, including cough, headache, rhinorrhoea and diarrhoea. There were no adverse effects of treatment on vital signs, ECG or physical findings. Importantly, the number of infections reported was similar in both the AZD5069 (nine patients) and placebo (eight patients) groups, and there was no apparent relationship with low neutrophil counts. It is of some concern that there were more adverse events leading to discontinuation in the AZD5069 treatment group (five (19.2\%) patients) than in the placebo group (none), and four out of these were related to exacerbation of bronchiectasis. However, a relationship between low neutrophil counts and development of infection cannot be ruled out. In a recent study of the $\mathrm{LTB}_{4}$-receptor antagonist BIIL 284, decreased pulmonary neutrophils correlated with increased bacterial burden in a murine model of P. aeruginosa lung infection [29]. However, the current study is too small to identify whether CXCR2 inhibition leads to an important increase in infection and whether this risk is confined to a definable group of patients. Larger and longer studies would be needed to address these issues. Interestingly, the patient who discontinued due to pneumonia had an increase in white blood cells during treatment with AZD5069, based on the leukocyte count at a study visit 4 days prior to hospitalisation and on admission to hospital $\left(5.6 \times 10^{9}\right.$ cells $\cdot \mathrm{L}^{-1}$ versus $10.1 \times 10^{9}$ cells $\cdot \mathrm{L}^{-1}$, respectively).

AZD5069 did not demonstrate any clinically relevant beneficial effects on sputum weight, lung function, TDI or symptoms as evaluated by the BronkoTest and SGRQ-C assessments. Few studies in bronchiectasis have shown any effect on HRQoL over a 28-day period, although longer studies of up to a year have shown positive effects of using macrolides in bronchiectasis. These studies have not shown significant differences in lung function [30, 31].

Demographic differences between the study drug and placebo groups at baseline were not thought likely to affect the primary outcome of the study. Although several patients in the study had blood neutrophil counts that dropped below the lower laboratory limit, no individual patient was withdrawn as a result of an a priori safety limit $\left(<1.0 \times 10^{9}\right.$ cells $\left.\cdot \mathrm{L}^{-1}\right)$ for neutropenia. A slight increase in peripheral blood platelet counts was observed. Although the cause is unknown, there is some evidence that CXCR2 antagonism and CXC chemokines such as IL-8 may affect megakaryocyte function [32,33]. Nevertheless, this change was not thought to be clinically relevant. 
Due to the small sample size of this study, it was not considered appropriate to stratify patients by more than two variables. As such, some differences between treatment groups emerged at baseline that were not accounted for (most notable were the differences in mean absolute neutrophil counts and disease duration), and so factors such as regression to the mean may have contributed to the observed effect. We chose to stratify by inhaled steroids as these were projected to be more prevalent in use than macrolides. In a larger study, stratifying for both ICS and macrolides would be considered, given that both may have an anti-inflammatory effect or have the potential to affect neutrophil biology. The small sample size also limited the sensitivity of the study to other outcomes; while the study was powered to detect differences in neutrophils in sputum, it was not designed or powered to detect clinical differences.

Although the size and duration of this study limit the conclusions that can currently be drawn on clinical outcomes, the results of the biomarker assays were intriguing. In the context of a reduction in inflammatory neutrophils in sputum, it might be expected that there would be a corresponding reduction in markers of inflammation, rather than the increases in sputum IL-6 and GRO- $\alpha$ that were observed [18]. We cannot exclude the possibility that these changes indicate local airways inflammation due to chronic infection. However, the absence of evidence of systemic inflammation as indicated by CRP and similar findings in normal volunteers treated with a higher dose of AZD5069 (100 mg twice daily) suggests that there may be an alternative explanation. The decrease of circulating neutrophils caused by CXCR2 blockade might cause an increase in blood levels of the natural ligands for CXCR2 receptors, IL-8 and GRO- $\alpha$, to partly compensate for the decrease in blood neutrophils or because of cytokine displacement/ build-up due to blocking of the receptor binding sites. The increased levels of these cytokines is unlikely to result in neutrophil activation as they were 100-1000 times lower than concentrations required to cause an induction of neutrophil effector functions as observed in vitro [34-36].

In the context of recent studies with other agents for the treatment of bronchiectasis, the reduction in sputum neutrophils observed in this study indicates some clinical potential for CXCR2 antagonism in the management of bronchiectasis [13, 30, 31, 37, 38]. Current treatment options such as long-term macrolide antibiotics and corticosteroids are either of limited effectiveness or have a number of potential drawbacks, such as development of bacterial resistance and adrenal suppression [13, 30, 31, 38, 39]. The CXC glutamine-leucine-arginine motif chemokines are central to the inflammatory response in bronchiectasis, COPD, asthma and other chronic inflammatory lung conditions $[9,15]$. A monoclonal antibody that selectively targeted only one of these chemokines (i.e. IL-8) proved clinically ineffective in patients with COPD [40]. A therapeutic approach, such as CXCR2 antagonism, that targets the activity of many of these chemokines may be more effective in bronchiectasis and other conditions characterised by neutrophilic inflammation. One difficulty is that these conditions are heterogeneous in respect to the pattern of lower airway inflammation and its likely cause. Nonmicrobial-driven neutrophilic inflammation due to damage-associated molecular patterns may be an important target for CXCR2 antagonists in bronchiectasis. Furthermore, CXCR2 antagonism may have additional beneficial effects on airway goblet cell hyperplasia and mucus production, independent of the modulation of neutrophil migration [15]. However, as with other novel approaches in development, larger studies of a longer duration with careful phenotyping of participants will be needed to determine the clinical utility of CXCR2 antagonism in bronchiectasis [37]. The concept that treatment responses to CXCR2 antagonism relate to the cause of neutrophilic airway inflammation is supported by a recent trial of another compound in patients with COPD showing an important reduction in exacerbation frequency in smokers but an increase in non- and ex-smokers [41].

\section{Conclusions}

AZD5069 was well tolerated and significantly reduced the absolute neutrophil cell count in spontaneous sputum from the bronchiectasis patients by $69 \%$ in this early-phase exploratory study. Larger, longer-term studies with careful phenotyping of patients with bronchiectasis and other conditions where neutrophilic bronchial inflammation is involved are required to determine whether this effect is associated with clinical benefit.

\section{Acknowledgements}

The authors would like to thank the participants, study site delivery teams and Kerstin Vikman and Kai Richter (AstraZeneca Mölndal, Sweden) for their contributions to this study. The authors also thank Mohib Uddin, Wendy Taylor, Anders Cavallin and Marleen Van Geest (Translational Science, iMED RIA, AstraZeneca) for their support of mediator analysis, data interpretation and discussions. We acknowledge the support of the National Institute for Health Research (NIHR) Comprehensive Local Research Network that supported recruitment in the UK after NIHR portfolio adoption and the Northern Ireland Respiratory Health Clinical Trials Network.

The study was conducted and managed by Quintiles (London, UK), a contract research organisation. Medical writing assistance was provided by David Candlish (inScience Communications, Springer Healthcare), and this was funded by AstraZeneca. 


\section{References}

1 Pasteur MC, Bilton D, Hill AT. British Thoracic Society guideline for non-CF bronchiectasis. Thorax 2010; 65: Suppl. 1, 1-58.

2 Tunney MM, Einarsson GG, Wei L, et al. The lung microbiota and bacterial abundance in patients with bronchiectasis when clinically stable and during exacerbation. Am J Respir Crit Care Med 2013; 187: 1118-1126.

3 Hill AT, Pasteur M, Cornford C, et al. Primary care summary of the British Thoracic Society Guideline on the management of non-cystic fibrosis bronchiectasis. Prim Care Respir J 2011; 20: 135-140.

4 European Respiratory Society. European Lung White Book: Editors' Introduction. www.erswhitebook.org/chapters/ editors-introduction/ Date last accessed: April 9, 2015.

5 Global Initiative for Asthma. Global Strategy for Asthma Management and Prevention. Global Initiative For Asthma, 2015.

6 Aujla SJ, Alcorn JF. $\mathrm{T}_{\mathrm{H}} 17$ cells in asthma and inflammation. Biochim Biophys Acta 2011; 1810: 1066-1079.

7 Woodruff PG, Kashayar R, Lazarus SC, et al. Relationship between airway inflammation, hyperresponsiveness, and obstruction in asthma. J Allergy Clin Immunol 2001; 108: 753-758.

8 Barnes PJ, Ito K, Adcock IM. Corticosteroid resistance in chronic obstructive pulmonary disease: inactivation of histone deacetylase. Lancet 2004; 363: 731-733.

9 Gernez Y, Tirouvanziam R, Chanez P. Neutrophils in chronic inflammatory airway diseases: can we target them and how? Eur Respir J 2010; 35: 467-469.

10 Kapur N, Bell S, Kolbe J, et al. Inhaled steroids for bronchiectasis. Cochrane Database Syst Rev 2009; 1: CD000996.

11 Kamath AV, Pavord ID, Ruparelia PR, et al. Is the neutrophil the key effector cell in severe asthma? Thorax 2005; 60: 529-530.

12 Balfour-Lynn IM, Lees B, Hall P, et al. Multicenter randomized controlled trial of withdrawal of inhaled corticosteroids in cystic fibrosis. Am J Respir Crit Care Med 2006; 173: 1356-1362.

13 Elborn JS, Tunney MM. Macrolides and bronchiectasis: clinical benefit with a resistance price. JAMA 2013; 309: 1295-1296.

14 De Soyza A, Brown JS, Loebinger MR, et al. Research priorities in bronchiectasis. Thorax 2013; 68: 695-696.

15 Chapman RW, Minnicozzi M, Celly CS, et al. A novel, orally active CXCR1/2 receptor antagonist, Sch527123, inhibits neutrophil recruitment, mucus production, and goblet cell hyperplasia in animal models of pulmonary inflammation. J Pharmacol Exp Ther 2007; 322: 486-493.

16 Traves SL, Smith SJ, Barnes PJ, et al. Specific CXC but not CC chemokines cause elevated monocyte migration in COPD: a role for CXCR2. J Leukoc Biol 2004; 76: 441-450.

17 Holz O, Khalilieh S, Ludwig-Sengpiel A, et al. SCH527123, a novel CXCR2 antagonist, inhibits ozone-induced neutrophilia in healthy subjects. Eur Respir J 2010; 35: 564-570.

18 O'Connor BJ, Leaker B, Barnes P, et al. Inhibition of LPS-induced neutrophilic inflammation in healthy volunteers. Eur Respir J 2007; 30: Suppl. 1, 209s.

19 Virtala R, Ekman AK, Jansson L, et al. Airway inflammation evaluated in a human nasal lipopolysaccharide challenge model by investigating the effect of a CXCR2 inhibitor. Clin Exp Allergy 2012; 42: 590-596.

20 Leaker B, Barnes PJ, O'Connor BJ. Inhibition of LPS-induced airway neutrophilic inflammation in healthy volunteers with an oral CXCR2 antagonist. Respir Res 2013; 14: 137.

21 Wilson CB, Jones PW, O'Leary CJ, et al. Validation of the St. George's Respiratory Questionnaire in bronchiectasis. Am J Respir Crit Care Med 1997; 156: 536-541.

22 Jones PW. St George's Respiratory Questionnaire Manual. London, Division of Cardiac and Vascular Science, St George's, University of London, 2009.

23 Wray H, Sparrow A. Safety and tolerability of single doses of AZD5069 in healthy volunteers. Eur Respir J 2011; 38: Suppl. 55, 3984.

24 Anthonisen NR, Manfreda J, Warren CPW, et al. Antibiotic therapy in exacerbations of chronic obstructive pulmonary disease. Ann Intern Med 1987; 106: 196-204.

25 Lichtman MA, Beutler E, Kaushansky K. Williams Hematology. 7th Edn. New York, McGraw-Hill Companies, 2006.

26 Elborn JS, Perrett J, Forsman-Semb K, et al. Efficacy, safety and effect on biomarkers of AZD9668 in cystic fibrosis. Eur Respir J 2012; 40: 969-976.

27 Wray H, Wilbraham D. Safety and tolerability of AZD5069 in healthy smokers following multiple ascending doses. Int J Immunorehab 2012; 14: 78.

28 Kirsten AM, Förster K, Radeczky E, et al. The safety and tolerability of twice-daily oral doses of AZD5069, a novel CXCR2 antagonist, in patients with moderate-to-severe COPD. Eur Respir J 2012; 40: Suppl. 56, 3083.

29 Döring G, Bragonzi A, Paroni M, et al. BIIL 284 reduces neutrophil numbers but increases P. aeruginosa bacteremia and inflammation in mouse lungs. J Cyst Fibros 2014; 13: 156-163.

30 Serisier DJ, Martin ML, McGuckin MA, et al. Effect of long-term, low-dose erythromycin on pulmonary exacerbations among patients with non-cystic fibrosis bronchiectasis: the BLESS randomized controlled trial. JAMA 2013; 309: 1260-1267.

31 Altenburg J, de Graaff CS, Stienstra Y, et al. Effect of azithromycin maintenance treatment on infectious exacerbations among patients with non-cystic fibrosis bronchiectasis: the BAT randomized controlled trial. JAMA 2013; 309: 1251-1259.

32 Emadi S, Clay D, Desterke C, et al. IL-8 and its CXCR1 and CXCR2 receptors participate in the control of megakaryocytic proliferation, differentiation, and ploidy in myeloid metaplasia with myelofibrosis. Blood 2005; 105: 464-473.

33 Gewirtz AM, Zhang J, Ratajczak J, et al. Chemokine regulation of human megakaryocytopoiesis. Blood 1995; 86: 2559-2567.

34 Glynn PC, Henney EM, Hall IP. Peripheral blood neutrophils are hyperresponsive to IL-8 and Gro- $\alpha$ in cryptogenic fibrosing alveolitis. Eur Respir J 2001; 18: 522-529.

35 Uddin M, Seumois G, Lau LC, et al. Enhancement of neutrophil function by the bronchial epithelium stimulated by epidermal growth factor. Eur Respir J 2008; 31: 714-724. 
Fu H, Bylund J, Karlsson A, et al. The mechanism for activation of the neutrophil NADPH-oxidase by the peptides formyl-Met-Leu-Phe and Trp-Lys-Tyr-Met-Val-Met differs from that for interleukin-8. Immunology 2004; 112: 201-210

37 Stockley R, De Soyza A, Gunawardena K, et al. Phase II study of a neutrophil elastase inhibitor (AZD9668) in patients with bronchiectasis. Respir Med 2013; 107: 524-533.

38 Wilson R, Welte T, Polverino E, et al. Ciprofloxacin dry powder for inhalation in non-cystic fibrosis bronchiectasis: a phase II randomised study. Eur Respir J 2013; 41: 1107-1115.

39 Holme J, Tomlinson JW, Stockley RA, et al. Adrenal suppression in bronchiectasis and the impact of inhaled corticosteroids. Eur Respir J 2008; 32: 1047-1052.

40 Mahler DA, Huang S, Tabrizi M, et al. Efficacy and safety of a monoclonal antibody recognizing interleukin-8 in COPD: a pilot study. Chest 2004; 126: 926-934.

41 Rennard S, Dale DC, Donohue JF, et al. CXCR2 Antagonist Mk-7123. A phase 2 proof-of-concept trial for chronic obstructive pulmonary disease. Am J Respir Crit Care Med 2013; 187: A6071. 